\title{
CORRELATION BETWEEN SPINOPELVIC PARAMETERS AND THE DEVELOPMENT OF LUMBAR DISC HERNIATION
}

\author{
๑ Cengiz TUNCER ${ }^{1}$, ๑ Ömer POLAT ${ }^{1}$, ๑ Uygur ER ${ }^{2}$ \\ ${ }^{1}$ Düzce University Faculty of Medicine, Department Neurosurgery, Düzce, Turkey \\ 2 Private Practice, Clinic of Neurosurgery, Ankara, Turkey
}

\begin{abstract}
Objective: The present study aimed to identify the correlation between spinopelvic parameters and the development of lumbar disc herniation, which is a condition usually surgically treated.

Materials and Methods: A total of 147 consecutive patients with low back pain were divided into two groups according to treatment with microdiscectomy or treatment with some medications. In all patients, pelvic incidence (PI), lumbar lordosis (LL), sacral slope (SS), and pelvic tilt (PT) angles were measured on standing profile roentgenograms of the lumbar spine and pelvis. Statistical differences were investigated between the two groups.

Results: The PI, LL, and SS values were significantly different between the two groups. However, the difference in terms of PT was not significant. Conclusion: Some spinopelvic parameters, such as PI, LL, SS, and PT, may be considered as predictive factors in the development of degenerative spinal diseases, and the restoration of sagittal balance may provide better results when these factors are considered.

Keywords: Lumbar disc herniation, lumbar lordosis, pelvic incidence, pelvic tilt, sacral slope
\end{abstract}

\section{INTRODUCTION}

Non-specific low back pain (LBP) affects people of all ages, and it is a significant cause of sick leaves worldwide ${ }^{(7)}$, with a lifetime incidence rate of approximately $30 \%{ }^{(4)}$. Lumbar disc herniation $(L D H)$ is one of the leading causes of $L B P^{(5)}$. However, lumbar microdiscectomy, which is a gold standard for surgical treatment of $\mathrm{LDH}$, is preferred in $<10 \%$ of patients with $\mathrm{LDH}^{(3)}$. Although the surgical indications of LDH are strictly defined, the development of $L D H$ requiring surgery may be identified using some different low back parameters. The structure of the spinopelvic complex may cause different degenerative spinal diseases, such as $\operatorname{LDH}^{(10)}$. The correlation between lumbar lordosis (LL), pelvic incidence (PI), sacral slope (SS), and pelvic tilt (PT), which are the most remarkable spinopelvic parameters, and LDH should be investigated. Thus, this retrospective study aimsto identify whether there is a correlation between these parameters and LDH, which needs surgery for treatment.

\section{MATERIALS AND METHODS}

\section{Patients}

This study included 147 consecutive patients with LBP who were admitted to our neurosurgery outpatient clinic. Informed consent was obtained from all participants. The inclusion criteria included being male or female patients with LBP who were aged between 25 and 65 years. After undergoing neurological examination and magnetic resonance imaging, some patients were diagnosed with LDH at only one level. Patients with diabetes mellitus or other types of metabolic disorders, such as hypo- or hyperthyroidism and metabolic bone disorders; those with uncontrolled hypertension, malignant diseases, osteoporosis, and previous spinal trauma/fracture; and those who previously underwent surgery in the lumbar region were excluded from the study. In addition, patients with a narrow spinal canal, spondylolisthesis, and any deformity in the spinal column were also excluded. Moreover, patients with a body mass index greater than $30.0 \mathrm{~kg} / \mathrm{m}^{2}$ were not included. Patients were divided into two groups as group I and group II. Patients with signs and symptoms that were indications for lumbar microdiscectomy (patients with neurologic deficit, difficulty in walking or urinary incontinence, or intractable pain after adequate physical therapy and medication) were included in group I. Meanwhile, those who did not meet the criteria for surgery (patients without neurologic deficit, difficulty in walking or urinary incontinence, but with herniation findings according to imaging studies) were included in group II. The PI, LL, SS, and PT angles of all the patients were measured on standing profile

Address for Correspondence: Uygur Er, Private Practice, Clinic of Neurosurgery, Ankara, Turkey Phone: +90 5055892355 E-mail: uygurer@gmail.com Received: 03.08.2019 Accepted: 14.09.2019 ORCID ID: orcid.org/0000-0002-6641-0075 
turkishspine

roentgenograms of the lumbar spine and pelvis. Measurements were performed as follows:

\section{Pelvic Incidence}

This parameter is used to characterize pelvic morphology and function ${ }^{(2)}$. PI is defined as the angle generated by the intersection of the line drawn from the center of the femoral heads to the middle of the sacral plate and the line passing perpendicular to the middle of the sacral plate ${ }^{(10)}$. PI is generally accepted as the angle that describes the relationship between the sacral plate and femoral heads. It is a stable morphologic parameter for each individual.

\section{Lumbar Lordosis}

$\mathrm{LL}$ is defined as the angle between the lines passing the $\mathrm{L} 1$ superior plate and sacral plate and is measured using the Cobb method on lateral lumbosacral roentgenogram. The present study compared the two groups in terms of PI and LL angles ${ }^{(9)}$.

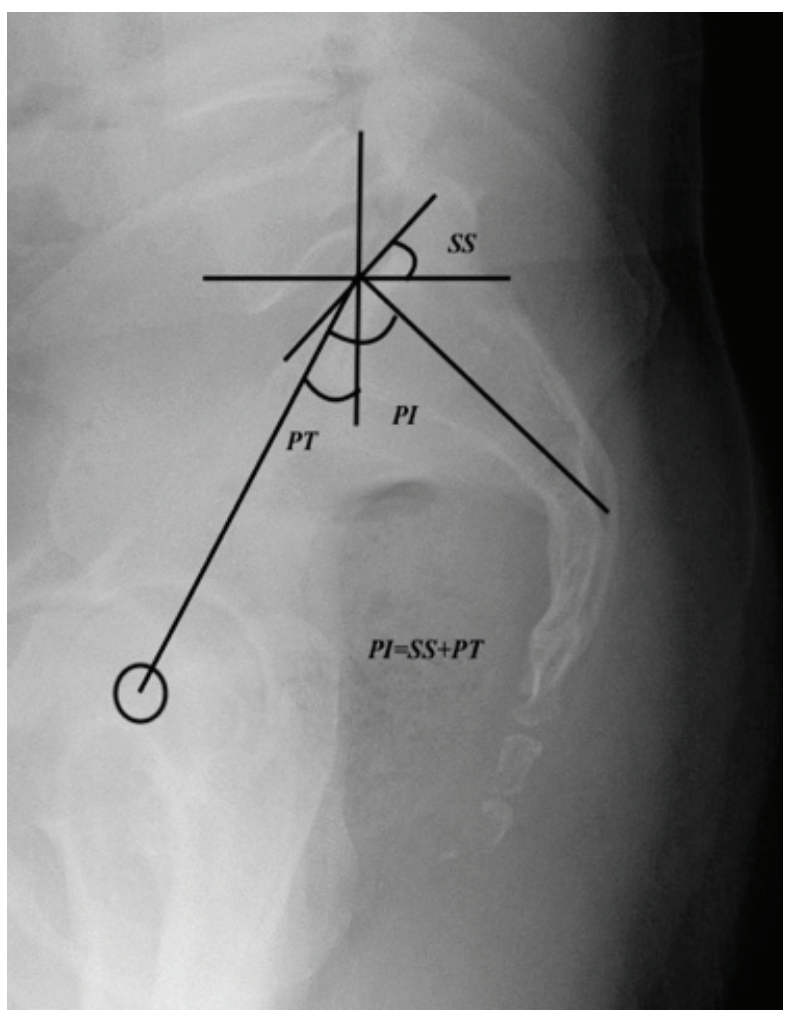

Figure 1. Spinopelvic parameters. The values obtained using the equation pelvic incidence=sacral slope+pelvic tilt can easily be proven on the figure geometrically

PI: Pelvic incidence, SS: Sacral slope, PT: Pelvic tilt

\section{Sacral Slope}

SS is one of the two spinopelvic parameters that define the orientation of the pelvis. The other parameter is PT. These two parameters are positional parameters. SS is defined as the angle between the sacral plate and horizontal line on standing lateral roentgenogram. A vertical sacrum is characterized by a low SS value, and a horizontal sacrum is characterized by a high SS value.

\section{Pelvic Tilt}

PT is defined as the angle between the line connecting the midpoint of the sacral plate to the bi-coxo-femoral axis and the vertical plane. The arithmetic equation between these parameters is as follows: $\mathrm{PI}=\mathrm{SS}+\mathrm{PT}$ (Figure 1$)^{(1)}$. Thus, if there is a significant difference between the two groups in terms of these parameters, it may be assessed via forward-looking estimations particularly in individuals who take part in the tail of the Gaussian distribution.

\section{Ethical Consideration}

All procedures involving human participants performed in this study were in accordance with the ethical standards of the institutional and/or national research committee and with the 1964 Declaration of Helsinki and its later amendments or comparable ethical standards.

\section{Statistical Analysis}

The Statistical Package for the Social Sciences software for Windows version 16.0 (IBM Corp., the USA) was used for statistical analyses. Intergroup comparisons of the data were performed using the independent t-test. Data were presented as mean \pm standard deviation. Any $p$ value less than 0.05 was considered as statistically significant.

\section{RESULTS}

The current study included 147 patients aged between 25 and 65 years with a mean age of $44.41 \pm 8.95$ years. Group I comprised 101 patients aged between 25 and 65 years with a mean age of $44.73 \pm 9.03$ years. Group II consisted of 46 patients aged between 25 and 65 years with a mean age of $43.70 \pm 8.81$ years. In terms of age, no significant difference was observed between the two groups ( $p>0.05)$. The male-tofemale $(M / F)$ ratio of the study population was 1.2 . The ratios in groups I and II were 1 and 1.9, respectively. A significant difference was observed between the two groups in terms of

Table 1. Pelvic incidence, lumbar lordosis, sacral slope and pelvic tilt values of groups I and II

\begin{tabular}{lllll}
\hline Spinopelvic parameters & Group I $(n=101)$ & Group II $(n=46)$ & Study population & $p$ \\
\hline PI & $54.63 \pm 8.24$ & $64.88 \pm 7.98$ & $57.84 \pm 9.43$ & $<0.005$ \\
\hline LL & $44.11 \pm 12.26$ & $50.56 \pm 10.62$ & $46.13 \pm 12.11$ & $<0.005$ \\
\hline SS & $30.22 \pm 7.52$ & $39.00 \pm 5.04$ & $33.00 \pm 7.98$ & $<0.005$ \\
\hline PT & $24.51 \pm 7.06$ & $25.80 \pm 6.30$ & $24.91 \pm 6.03$ & $<0.005$ \\
\hline
\end{tabular}

PI: Pelvic incidence, LL: Lumbar lordosis, SS: Sacral slope, PT: Pelvic tilt, numbers are mean \pm standard deviation 
gender distribution $(\mathrm{p}<0.05)$. The mean $\mathrm{Pl}$ value of the study population was $57.84^{\circ} \pm 9.43^{\circ}$, ranging from $36^{\circ}$ to $77^{\circ}$. The mean PI value of group I was $54.63^{\circ} \pm 8.24^{\circ}$, and that of group II was $64.88^{\circ} \pm 7.98^{\circ}$. A significant difference was observed between the two groups in terms of PI value $(p<0.05)$.

The mean $L L$ value of the study population was $46.13^{\circ} \pm 12.11^{\circ}$, ranging from $6^{\circ}$ to $68^{\circ}$. The mean LL value of group I was $44.11^{\circ} \pm 12.26^{\circ}$, and that of group II was $50.56^{\circ} \pm 10.62^{\circ}$. A significant difference was observed between the two groups in terms of LL value $(p<0.05)$. The mean SS value of the study population was $33.00^{\circ} \pm 7.98^{\circ}$, ranging from $14^{\circ}$ to $49^{\circ}$. The mean SS value of group I was $30.22^{\circ} \pm 7.52^{\circ}$, and that of group II was $39.00^{\circ} \pm 5.04^{\circ}$. A significant difference was also revealed between the two groups in terms of SS value $(p<0.05)$. The mean PT value of the study population was $24.91^{\circ} \pm 6.03^{\circ}$, ranging from $5^{\circ}$ to $42^{\circ}$. The mean PT value of group I was $24.51^{\circ} \pm 7.06^{\circ}$, and that of group II was $25.80^{\circ} \pm 6.30^{\circ}$. No significant difference was found between the two groups in terms of PT value $(p>0.05)$, (Table 1). In conclusion, statistically significant differences were observed in PI, LL, and SS values, but not in PT value.

\section{DISCUSSION}

Standing in erect position is a basic human attribute. As bipedal locomotion has developed in the history of transformation from Hominidae to erect human species ${ }^{(10)}$, it causes some spine problems in humans. LDH develops as a result of degenerative process ${ }^{(8)}$. Degenerative diseases are more likely to occur in an unbalanced loading and under asymmetric force vectors. Spinopelvic parameters may be helpful in identifying the risk factors for lumbar degenerative disc disease, and such parameters can be used to explain why asymmetric loading causes LDH. PI and LL are the most important spinopelvic parameters that form the lumbo-pelvic shape. The International Spine Study Group has recently reported about PI-LL mismatch ${ }^{(11)}$. According to their interpretation, PI-LL mismatch is correlated to disability. In relation to this reason, these two parameters were used in this study to identify the correlation between the development of LDH and spinopelvic morphology. By contrast, two other parameters defining the orientation of the pelvis, SS, and PT were also investigated for their possible correlation with the development of LDH. PI is a morphologic constant parameter and is not affected by posture or pelvic position ${ }^{(1)}$. Therefore, degenerative lumbar lesions are expected not to influence PI value. LL is another important parameter that forms the lumbar sagittal shape and even affects the global sagittal balance. LL angle is of clinical interest in assessing lumbar alignment ${ }^{(6)}$. Thus, the influences of these two parameters in developing degenerative disc pathologies is worth investigating. At the same time, a weak correlation was observed between PI and LL, which strengthens this thought. This study showed a negative correlation between PI value and the development of LDH. The same negative correlation was observed between LL and SS values and the development of
LDH. However, these statistical results are applicable in patients with LBP. These correlations may only show the severity of the situation. In this study, we cannot conclude that individuals with high PI, LL, or SS values do not develop LDH. However, we can say that individuals with existing or previously diagnosed LDH who have low PI, LL, and SS values are more likely to undergo surgery. Based on gender differences, men with LBP were less likely to undergo surgery compared to women with standard indications of LDH. However, the M/F ratio of group I was 1 , which makes this result challenging to interpret. $L L$ is the important parameter that may explain this result. However, no significant differences were observed between men and women in terms of $L L$ values. The influences of pelvic shape and orientation may help to identify individuals who are at risk of developing LDH at some degree. At the same time, the restoration of these parameters, except $\mathrm{PI}$, may help surgeons establish a preoperative plan. The sagittal balance of the spine should be considered during the evaluation of each patient with degenerative spine diseases. The restoration of sagittal balance with the main treatment of any pathology of the spine is crucial in obtaining good outcome.

\section{Study Limitations}

The current study had some limitations. First, the use of the LL angle alone may not be sufficient. There are different types of LL and each type representing a corresponding PI value may have different clinic outcomes ${ }^{(10,12)}$. Second, this is a retrospective study performed on patients with LBP. Thus, orthopedic spine surgeons must conduct prospective studies about this subject in real-life settings.

\section{CONCLUSION}

Some spinopelvic parameters, such as PI, LL, SS, and PT, may be considered as predictive factors for the development of degenerative spinal diseases, and the restoration of sagittal balance with consideration of these parameters may provide better results. Moreover, $\mathrm{PI}$ is a constant structural parameter; thus, an individual with low PI who presents with LBP must be monitored to prevent the development of LDH.

Acknowledgements: The preparation for the publication of this article was partly supported by the Turkish Neurosurgical Society.

\section{Ethics}

Ethics Committee Approval: Retrospective analysis in patients' savings. It doesn't need Ethics Committee approval.

Informed Consent: Written informed consent was obtained from all participants.

Peer-review: Internally peer-reviewed.

\section{Authorship Contributions}

Surgical and Medical Practices: U.E., C.T., Ö.P., Concept: C.T., Design: U.E., Data Collection or Processing: Ö.P., C.T., Analysis or Interpretation: U.E., Literature Search: Ö.P., C.T., Writing: U.E. 
turkishspine

Conflict of Interest: No conflict of interest was declared by the authors.

Financial Disclosure: The authors declared that this study received no financial support

\section{REFERENCES}

1. Barrey C, Jund J, Noseda O, Roussouly P. Sagittal balance of the pelvisspine complex and lumbar degenerative diseases. A comparative study about 85 cases. Eur Spine J 2007;16:1459-67.

2. Duval-Beaupére G, Legaye J. A barycentremetric study of the sagittal shape of the spine and pelvis: the conditions required for an economic standing position. Ann Biomed Eng 1992;20:451-62.

3. Gibson JN, Waddel G. Surgical interventions for lumbar disc prolapse. Cochrane Database Syst Rev 2007; CD001350.

4. Gugliotta M, da Costa BR, Dabis E, Theiler R, Juni P, Reichenbach S, et al. Surgical versus conservative treatment for lumbar disc herniation: A prospective cohort study. BMJ Open 2016;6:e012938.

5. Konstantinou K, Dunn KM. Sciatica: review of epidemiological studies and prevalence estimates. Spine (Phila Pa 1976) 2008;33:2464-72.
6. Laird RA, Kent P, Keating JL. How consistent are lordosis, range of movement and lumbo-pelvic rhythm in people with and without back pain? BMC Musculoskeletal Disord 2016;17:403-17.

7. Maher C, Underwood M, Buchbinder R. Non-specific low back pain. Lancet 2017;389:736-47.

8. Mardare M, Oprea M, Popa I, Zazgyva A, Niculescu M, Poenaru DV. Sagittal balance parameters correlate with spinal conformational type and MRI changes in lumbar degenerative disc disease: results of a retrospective study. Eur J Orthop Trauma 2016;26:735-43.

9. Polly DW, Kilkelly FX, McHale KA, Asplund LM. Measurement of lumbar lordosis. Spine 1996;21:1530-6.

10. Roussouly P, Pinheiro-Franco JL. Biomechanical analysis of the spino-pelvic organization and adaptation in pathology. Eur Spine J 2011;20:S609-S15.

11. Schwab Fl, Blondel B, Bess S, Hostin R, Shaffrey Cl, Smith JS, et al. Radiographical spinopelvic parameters and disability in the setting of adult spinal deformity: a prospective multicenter analysis. Spine (Phila PA 1976) 2013;38:E803-E12.

12. Tono O, Hasegawa K, Okamoto M, Hatsushikano S, Shimoda $H$, Watanabe K, et al. Lumbar lordosis doesn't correlate with pelvic incidence in the cases with the lordosis apex located at L3 or above. Eur Spine J 2018;1-7. 